\title{
Firefly Algorithm, Stochastic Test Functions and Design Optimisation
}

\author{
Xin-She Yang \\ Department of Engineering, University of Cambridge, \\ Trumpington Street, Cambridge CB2 1PZ, UK \\ Email: xy227@cam.ac.uk
}

March 9, 2010

\begin{abstract}
Modern optimisation algorithms are often metaheuristic, and they are very promising in solving NP-hard optimization problems. In this paper, we show how to use the recently developed Firefly Algorithm to solve nonlinear design problems. For the standard pressure vessel design optimisation, the optimal solution found by FA is far better than the best solution obtained previously in literature. In addition, we also propose a few new test functions with either singularity or stochastic components but with known global optimality, and thus they can be used to validate new optimisation algorithms. Possible topics for further research are also discussed.
\end{abstract}

To cite this paper as follows: Yang, X. S., (2010) 'Firefly Algorithm, Stochastic Test Functions and Design Optimisation', Int. J. Bio-Inspired Computation, Vol. 2, No. 2, pp.78-84.

\section{Introduction}

Most optimization problems in engineering are nonlinear with many constraints. Consequently, to find optimal solutions to such nonlinear problems requires efficient optimisation algorithms (Deb 1995, Baeck et al 1997, Yang 2005). In general, optimisation algorithms can be classified into two main categories: deterministic and stochastic. Deterministic algorithms such as hill-climbing will produce the same set of solutions if the iterations start with the same initial guess. On the other hand, stochastic algorithms often produce different solutions even with the same initial starting point. However, the final results, though slightly different, will usually converge to the same optimal solutions within a given accuracy.

Deterministic algorithms are almost all local search algorithms, and they are quite efficient in finding local optima. However, there is a risk for the algorithms to be trapped at local optima, while the global optima are out of reach. A common practice is to introduce some stochastic component to an algorithm so that it becomes possible to jump out of such locality. In this case, algorithms become stochastic.

Stochastic algorithms often have a deterministic component and a random component. The stochastic component can take many forms such as simple randomization by randomly sampling the search space or by random walks. Most stochastic algorithms can be considered as metaheuristic, and good examples are genetic algorithms (GA) (Holland 1976, Goldberg 1989) and particle swarm optimisation (PSO) (Kennedy and Eberhart 1995, Kennedy et al 2001). Many modern metaheuristic algorithms were developed based on the swarm intelligence in nature (Kennedy and Eberhart 1995, Dorigo and Stützle 2004). New modern metaheuristic algorithms are being developed and begin to show their power and efficiency. For example, the Firefly Algorithm developed by the author shows its superiority over some traditional algorithms (Yang 2009, Yang 2009, Lukasik and Zak 2009).

The paper is organized as follows: we will first briefly outline the main idea of the Firefly Algorithm in Section 2, and we then describe a few new test functions with singularity and/or 
randomness in Section 3. In Section 4, we will use FA to find the optimal solution of a pressure vessel design problem. Finally, we will discuss the topics for further studies.

\section{Firefly Algorithm and its Implementation}

\subsection{Firefly Algorithm}

The Firefly Algorithm was developed by the author (Yang 2008, Yang 2009), and it was based on the idealized behaviour of the flashing characteristics of fireflies. For simplicity, we can idealize these flashing characteristics as the following three rules

- all fireflies are unisex so that one firefly is attracted to other fireflies regardless of their sex;

- Attractiveness is proportional to their brightness, thus for any two flashing fireflies, the less brighter one will move towards the brighter one. The attractiveness is proportional to the brightness and they both decrease as their distance increases. If no one is brighter than a particular firefly, it moves randomly;

- The brightness or light intensity of a firefly is affected or determined by the landscape of the objective function to be optimised.

For a maximization problem, the brightness can simply be proportional to the objective function. Other forms of brightness can be defined in a similar way to the fitness function in genetic algorithms or the bacterial foraging algorithm (BFA) (Gazi and Passino 2004).

In the FA, there are two important issues: the variation of light intensity and formulation of the attractiveness. For simplicity, we can always assume that the attractiveness of a firefly is determined by its brightness or light intensity which in turn is associated with the encoded objective function. In the simplest case for maximum optimization problems, the brightness $I$ of a firefly at a particular location $\mathbf{x}$ can be chosen as $I(\mathbf{x}) \propto f(\mathbf{x})$. However, the attractiveness $\beta$ is relative, it should be seen in the eyes of the beholder or judged by the other fireflies. Thus, it should vary with the distance $r_{i j}$ between firefly $i$ and firefly $j$. As light intensity decreases with the distance from its source, and light is also absorbed in the media, so we should allow the attractiveness to vary with the degree of absorption.

In the simplest form, the light intensity $I(r)$ varies with the distance $r$ monotonically and exponentially. That is

$$
I=I_{0} e^{-\gamma r},
$$

where $I_{0}$ is the original light intensity and $\gamma$ is the light absorption coefficient. As a firefly's attractiveness is proportional to the light intensity seen by adjacent fireflies, we can now define the attractiveness $\beta$ of a firefly by

$$
\beta=\beta_{0} e^{-\gamma r^{2}},
$$

where $\beta_{0}$ is the attractiveness at $r=0$. It is worth pointing out that the exponent $\gamma r$ can be replaced by other functions such as $\gamma r^{m}$ when $m>0$. Schematically, the Firefly Algorithm (FA) can be summarised as the pseudo code. 


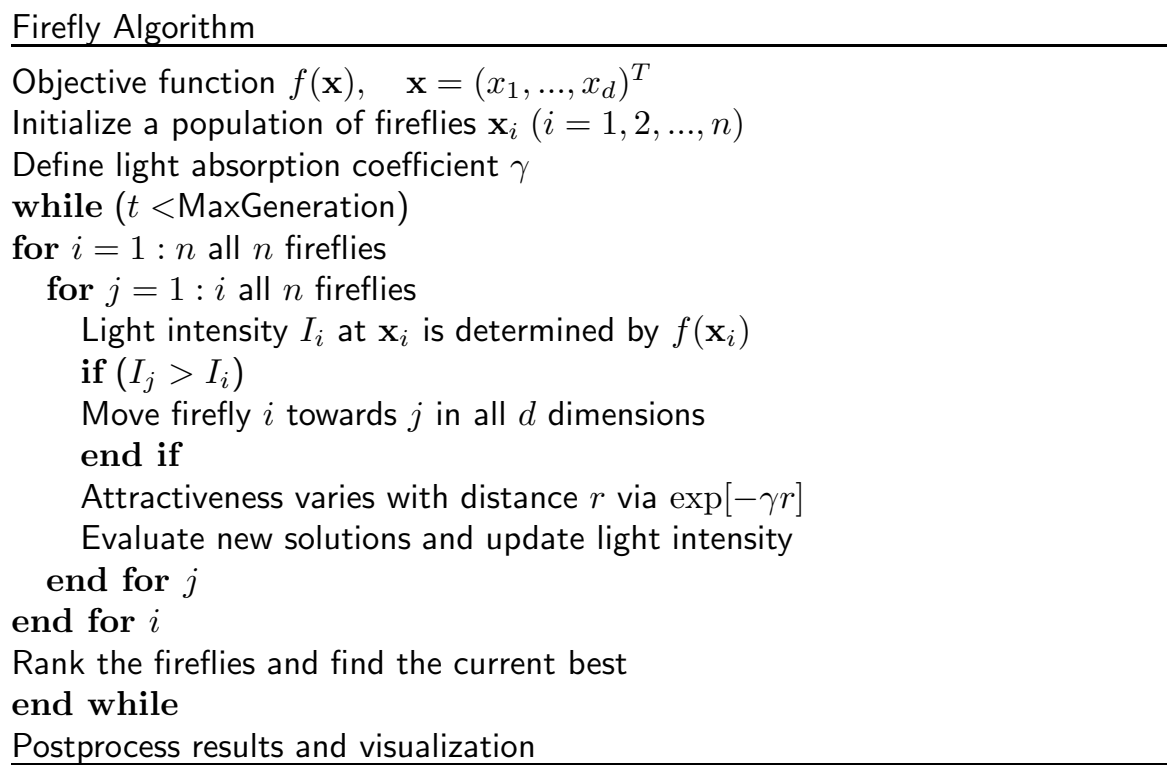

The distance between any two fireflies $i$ and $j$ at $\mathbf{x}_{i}$ and $\mathbf{x}_{j}$ can be the Cartesian distance $r_{i j}=\left\|\mathbf{x}_{i}-\mathbf{x}_{j}\right\|_{2}$ or the $\ell_{2}$-norm. For other applications such as scheduling, the distance can be time delay or any suitable forms, not necessarily the Cartesian distance.

The movement of a firefly $i$ is attracted to another more attractive (brighter) firefly $j$ is determined by

$$
\mathbf{x}_{i}=\mathbf{x}_{i}+\beta_{0} e^{-\gamma r_{i j}^{2}}\left(\mathbf{x}_{j}-\mathbf{x}_{i}\right)+\alpha \boldsymbol{\epsilon}_{i},
$$

where the second term is due to the attraction, while the third term is randomization with the vector of random variables $\boldsymbol{\epsilon}_{i}$ being drawn from a Gaussian distribution.

For most cases in our implementation, we can take $\beta_{0}=1, \alpha \in[0,1]$, and $\gamma=1$. In addition, if the scales vary significantly in different dimensions such as $-10^{5}$ to $10^{5}$ in one dimension while, say, $-10^{-3}$ to $10^{3}$ along others, it is a good idea to replace $\alpha$ by $\alpha S_{k}$ where the scaling parameters $S_{k}(k=1, \ldots, d)$ in the $d$ dimensions should be determined by the actual scales of the problem of interest.

In essence, the parameter $\gamma$ characterizes the variation of the attractiveness, and its value is crucially important in determining the speed of the convergence and how the FA algorithm behaves. In theory, $\gamma \in[0, \infty)$, but in practice, $\gamma=O(1)$ is determined by the characteristic/mean length $S_{k}$ of the system to be optimized. In one extreme when $\gamma \rightarrow 0$, the attractiveness is constant $\beta=\beta_{0}$. This is equivalent to saying that the light intensity does not decrease in an idealized sky. Thus, a flashing firefly can be seen anywhere in the domain. Thus, a single (usually global) optimum can easily be reached. This corresponds to a special case of particle swarm optimization (PSO). In fact, if the inner loop for $j$ is removed and $I_{j}$ is replaced by the current global best $\mathbf{g}_{*}$, FA essentially becomes the standard PSO, and, subsequently, the efficiency of this special case is the same as that of PSO. On the other hand, if $\gamma \rightarrow \infty$, we have $\beta(r) \rightarrow \delta(r)$, which is a Dirac $\delta$-function. This means that the attractiveness is almost zero in the sight of other fireflies, or the fireflies are short-sighted. This is equivalent to the case where the fireflies fly in a very foggy region randomly. No other fireflies can be seen, and each firefly roams in a completely random way. Therefore, this corresponds to the completely random search method. So $\gamma$ partly controls how the algorithm behaves. It is also possible to adjust $\gamma$ so that multiple optima can be found at the same during iterations.

\section{$2.2 \quad$ Numerical Examples}

From the pseudo code, it is relatively straightforward to implement the Firefly Algorithm using a popular programming language such as Matlab. We have tested it against more than a dozen test 


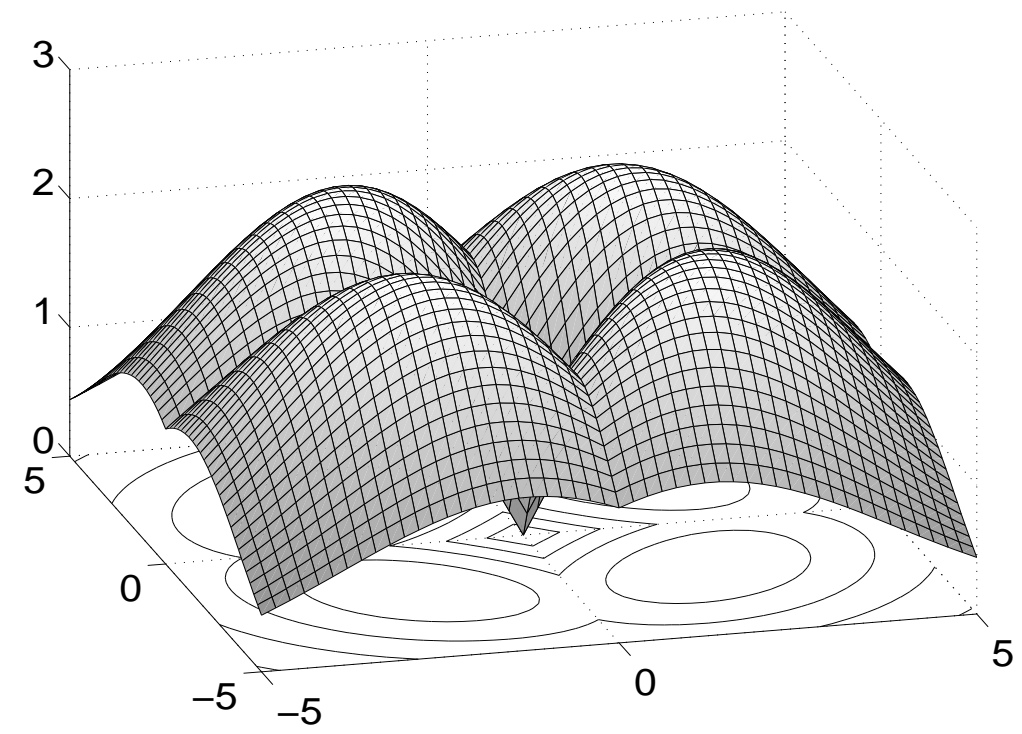

Figure 1: Four global maxima at $( \pm 1 / 2, \pm 1 / 2)$.

functions such as the Ackley function

$$
\begin{aligned}
& f(\mathbf{x})=-20 \exp \left[-\frac{1}{5} \sqrt{\frac{1}{d} \sum_{i=1}^{d} x_{i}^{2}}\right] \\
& -\exp \left[\frac{1}{d} \sum_{i=1}^{d} \cos \left(2 \pi x_{i}\right)\right]+20+e
\end{aligned}
$$

which has a unique global minimum $f_{*}=0$ at $(0,0, \ldots, 0)$. From a simple parameter studies, we concluded that, in our simulations, we can use the following values of parameters $\alpha=0.2, \gamma=1$, and $\beta_{0}=1$. As an example, we now use the FA to find the global maxima of the following function

$$
f(\mathbf{x})=\left(\sum_{i=1}^{d}\left|x_{i}\right|\right) \exp \left(-\sum_{i=1}^{d} x_{i}^{2}\right),
$$

with the domain $-10 \leq x_{i} \leq 10$ for all $(i=1,2, \ldots, d)$ where $d$ is the number of dimensions. This function has multiple global optima. In the case of $d=2$, we have 4 equal maxima $f_{*}=1 / \sqrt{e} \approx$ 0.6065 at $(1 / 2,1 / 2),(1 / 2,-1 / 2),(-1 / 2,1 / 2)$ and $(-1 / 2,-1 / 2)$ and a unique global minimum at $(0,0)$.

The four peaks are shown in Fig. 1, and these global maxima can be found using the implemented Firefly Algorithms after about 500 function evaluations. This corresponds to 25 fireflies evolving for 20 generations or iterations. The initial locations of 25 fireflies are shown Fig. 2 and their final locations after 20 iterations are shown in Fig. 3. We can see that the Firefly Algorithm is very efficient. Recently studies also confirmed its promising power in solving nonlinear constrained optimization tasks (Yang 2009, Lukasik and Zak 2009).

\section{New Test Functions}

The literature about test functions is vast, often with different collections of test functions for validating new optimisation algorithms. Test functions such as Rosenbrock's banana function and Ackley's function mentioned earlier are well-known in the optimisation literature. Almost all these 


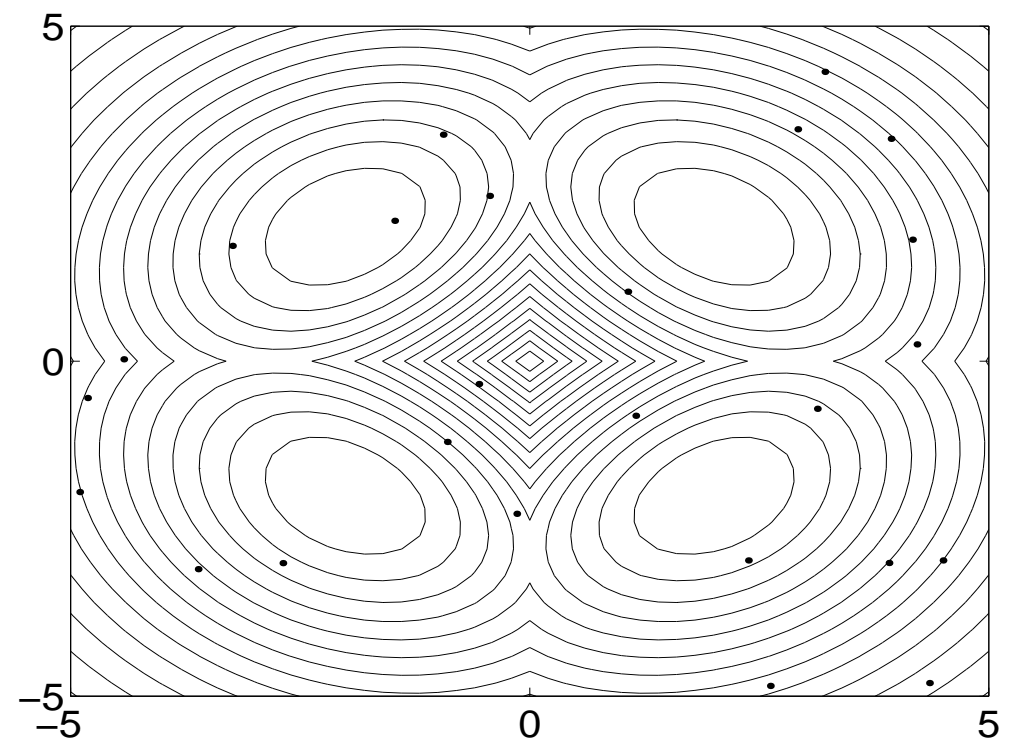

Figure 2: Initial locations of 25 fireflies.

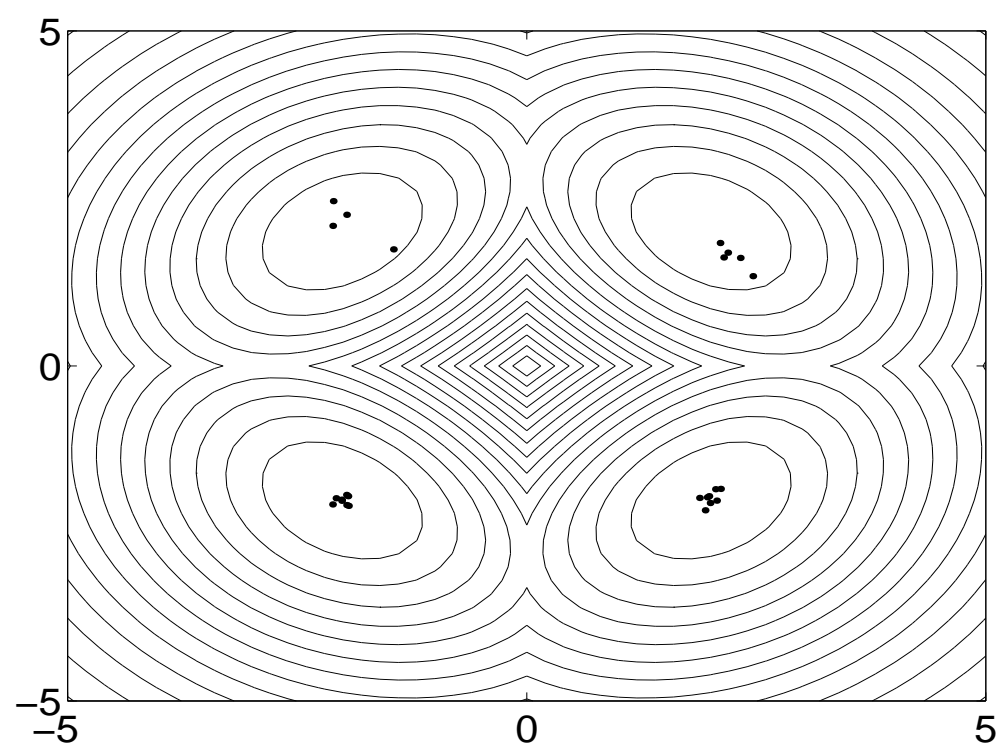

Figure 3: Final locations after 20 iterations. 
test functions are deterministic and smooth. In the rest of this paper, we first propose a few new test functions which have some singularity and/or stochastic components. Some of the formulated functions have stochastic components but their global optima are deterministic. Then, we will use the Firefly Algorithm to find the optimal solutions of some of these new functions.

The first test function we have designed is a multimodal nonlinear function

$$
\begin{gathered}
f(\mathbf{x})=\left[e^{-\sum_{i=1}^{d}\left(x_{i} / \beta\right)^{2 m}}-2 e^{-\sum_{i=1}^{d}\left(x_{i}-\pi\right)^{2}}\right] \\
\cdot \prod_{i=1}^{d} \cos ^{2} x_{i}, \quad m=5,
\end{gathered}
$$

which looks like a standing-wave function with a defect (see Fig. 4). It has many local minima and the unique global minimum $f_{*}=-1$ at $\mathbf{x}_{*}=(\pi, \pi, \ldots, \pi)$ for $\beta=15$ within the domain $-20 \leq x_{i} \leq 20$ for $i=1,2, \ldots, d$. By using the Firefly Algorithm with 20 fireflies, it is easy to find the global minimum in just about 15 iterations. The results are shown in Fig. 5 and Fig. 6.

As most test functions are smooth, the next function we have formulated is also multimodal but it has a singularity

$$
f(\mathbf{x})=\left(\sum_{i=1}^{d}\left|x_{i}\right|\right) \cdot \exp \left[-\sum_{i=1}^{d} \sin \left(x_{i}^{2}\right)\right],
$$

which has a unique global minimum $f_{*}=0$ at $\mathbf{x}_{*}=(0,0, \ldots, 0)$ in the domain $-2 \pi \leq x_{i} \leq 2 \pi$ where $i=1,2, \ldots, d$. At a first look, this function has some similarity with function (5) discussed earlier. However, this function is not smooth, and its derivatives are not well defined at the optimum $(0,0, \ldots, 0)$. The landscape of this forest-like function is shown in Fig. 7 and its $2 \mathrm{D}$ contour is displayed in Fig. 8.

Almost all existing test functions are deterministic. Now let us design a test function with stochastic components

$$
\begin{aligned}
& f(x, y)=-5 e^{-\beta\left[(x-\pi)^{2}+(y-\pi)^{2}\right]} \\
& -\sum_{j=1}^{K} \sum_{i=1}^{K} \epsilon_{i j} e^{-\alpha\left[(x-i)^{2}+(y-j)^{2}\right]},
\end{aligned}
$$

where $\alpha, \beta>0$ are scaling parameters, which can often be taken as $\alpha=\beta=1$. Here the random variables $\epsilon_{i j}(i, j=1, \ldots, K)$ obey a uniform distribution $\epsilon_{i j} \sim \operatorname{Unif}[0,1]$. The domain is $0 \leq x, y \leq K$ and $K=10$. This function has $K^{2}$ local valleys at grid locations and the fixed global minimum at $\mathbf{x}_{*}=(\pi, \pi)$. It is worth pointing that the minimum $f_{\min }$ is random, rather than a fixed value; it may vary from $-\left(K^{2}+5\right)$ to -5 , depending $\alpha$ and $\beta$ as well as the random numbers drawn.

For stochastic test functions, most deterministic algorithms such as hill-climbing would simply fail due to the fact that the landscape is constantly changing. However, metaheuristic algorithms could still be robust in dealing with such functions. The landscape of a realization of this stochastic function is shown in Fig. 9.

Using the Firefly Algorithm, we can find the global minimum in about 15 iterations for $n=20$ fireflies. That is, the total number of function evaluations is just 300. This is indeed very efficient and robust. The initial locations of the fireflies are shown in Fig. 10 and the final results are shown Fig.11.

Furthermore, we can also design a relative generic stochastic function which is both stochastic and non-smooth

$$
f(\mathbf{x})=\sum_{i=1}^{d} \epsilon_{i}\left|x_{i}\right|^{i}, \quad-5 \leq x_{i} \leq 5,
$$

where $\epsilon_{i}(i=1,2, \ldots, d)$ are random variables which are uniformly distributed in $[0,1]$. That is, $\epsilon_{i} \sim \operatorname{Unif}[0,1]$. This function has the unique minimum $f_{*}=0$ at $\mathbf{x}_{*}=(0,0, \ldots, 0)$ which is also singular.

We found that for most problems $n=15$ to 50 would be sufficient. For tougher problems, larger $n$ can be used, though excessively large $n$ should not be used unless there is no better alternative, as it is more computationally extensive for large $n$. 


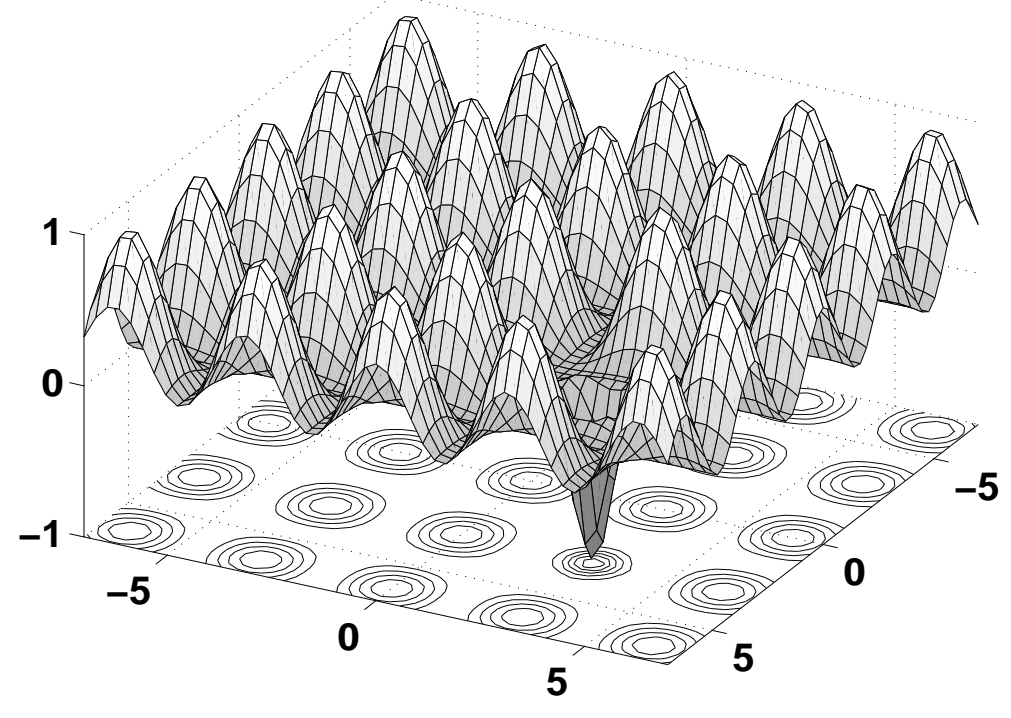

Figure 4: The standing wave function for two independent variables with the global minimum $f_{*}=-1$ at $(\pi, \pi)$.

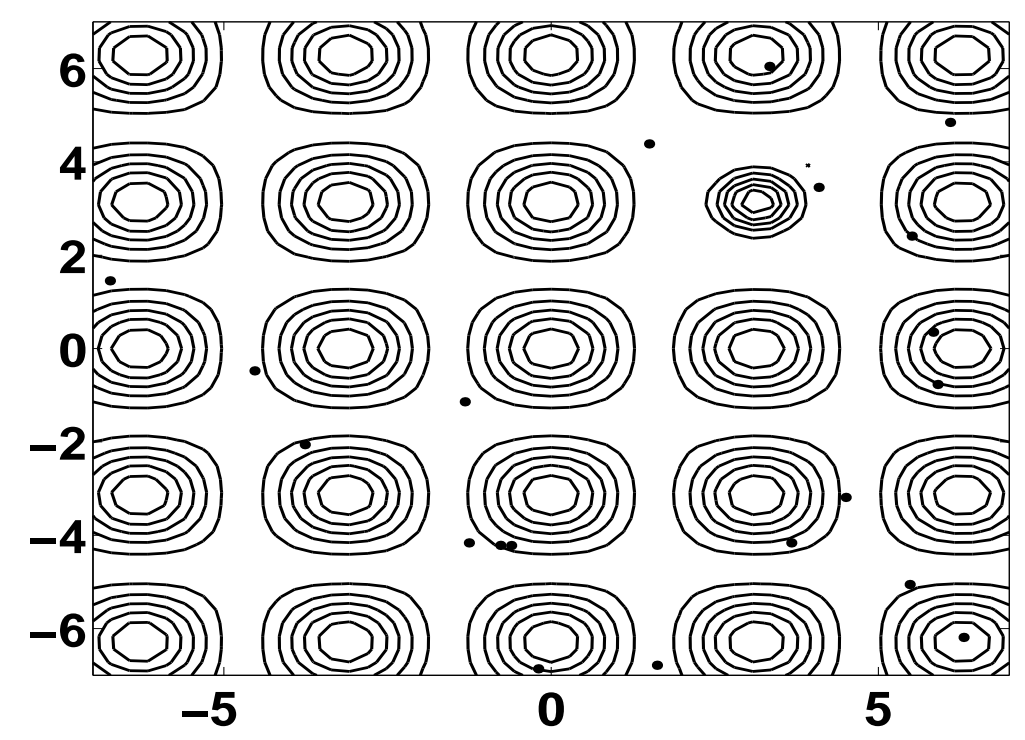

Figure 5: The initial locations of 20 fireflies. 


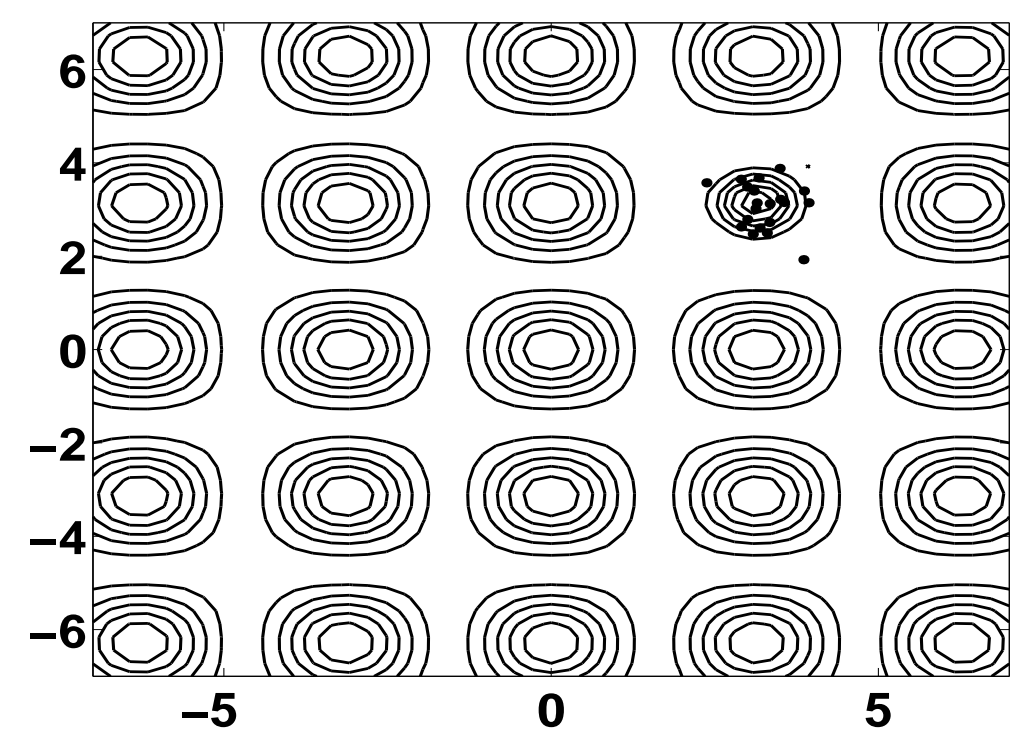

Figure 6: Final locations after 15 iterations.

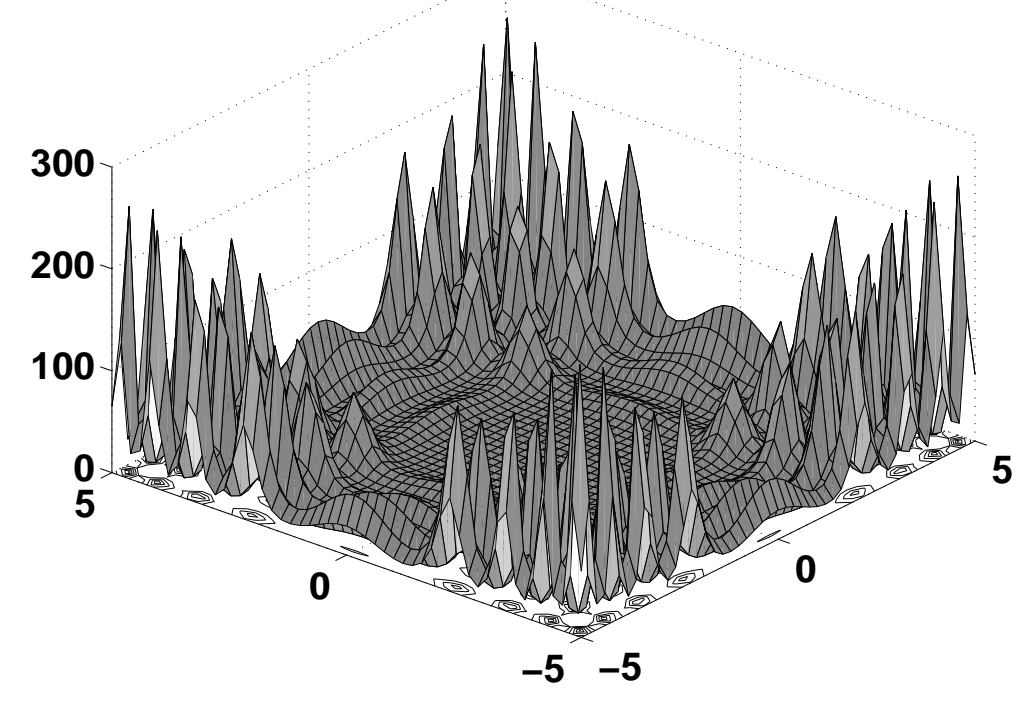

Figure 7: The landscape of function (7). 


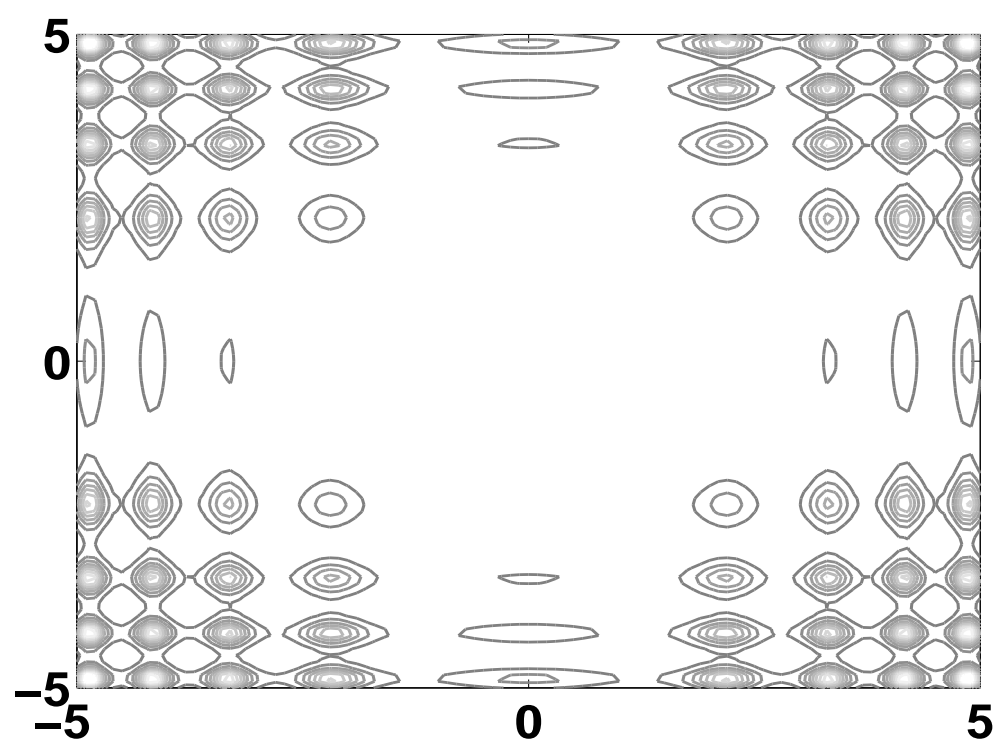

Figure 8: Contour of function (7).

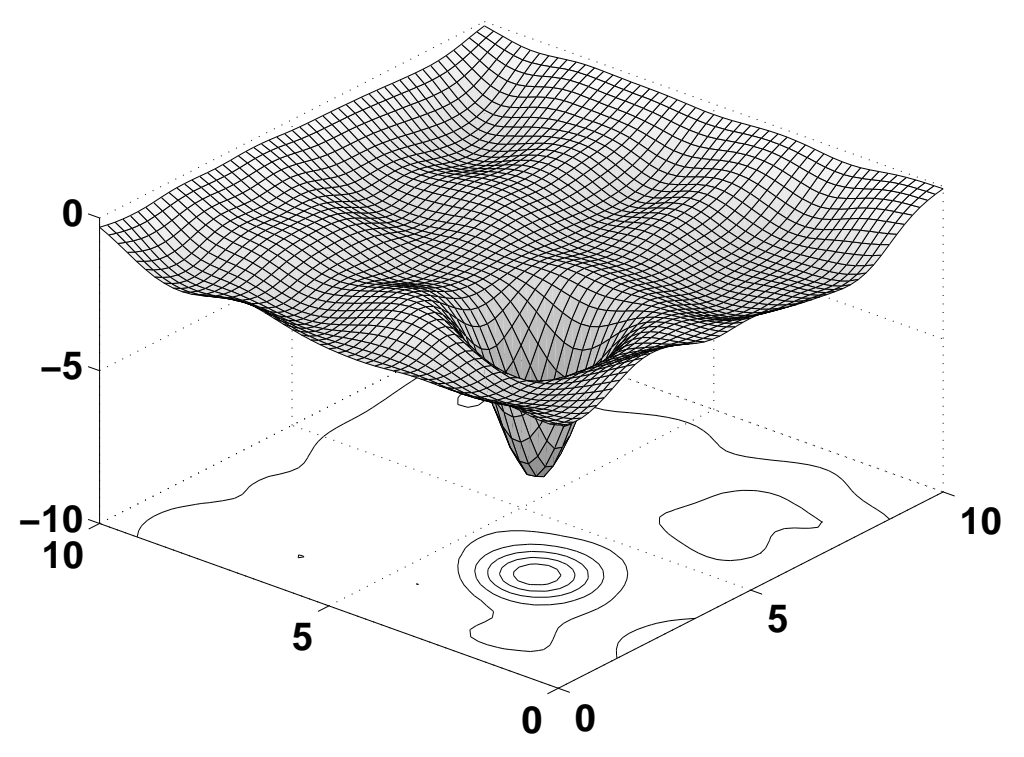

Figure 9: The 2D Stochastic function for $K=10$ with a unique global minimum at $(\pi, \pi)$, though the value of this global minimum is somewhat stochastic. 


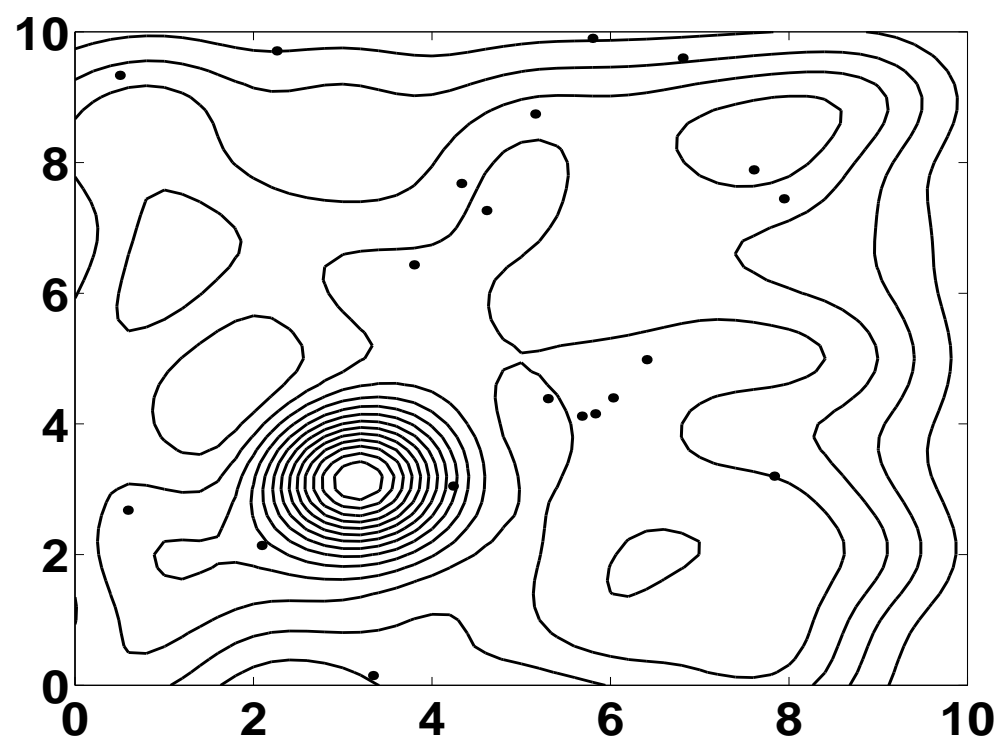

Figure 10: The initial locations of 20 fireflies.

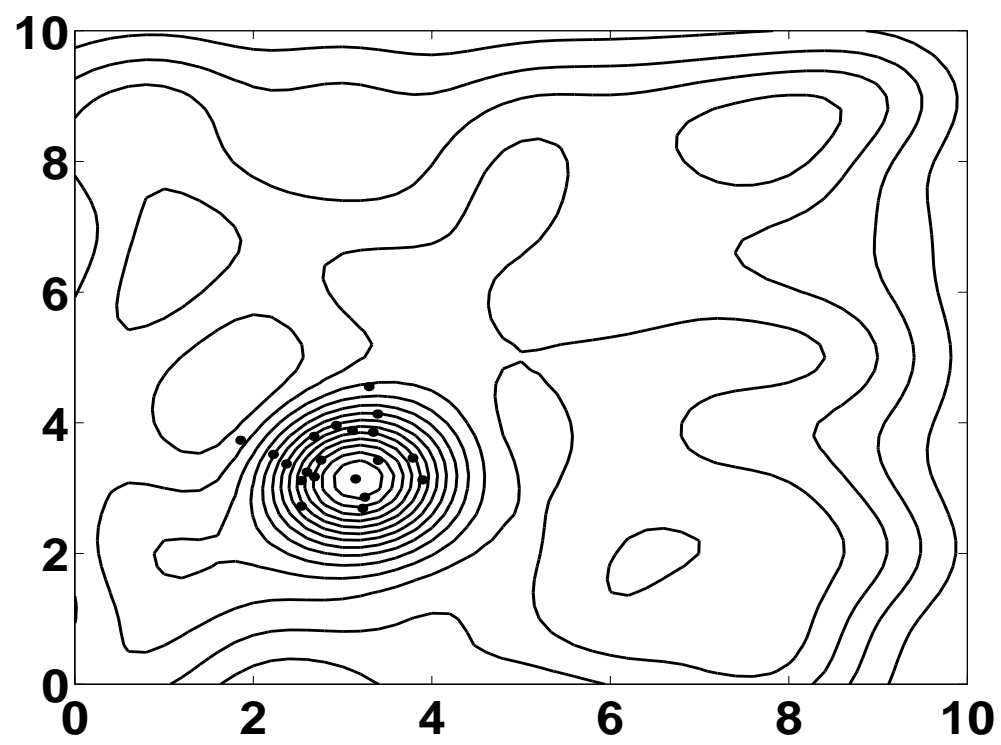

Figure 11: The final locations of 20 fireflies after 15 iterations, converging into $(\pi, \pi)$. 


\section{Engineering Applications}

Now we can apply the Firefly Algorithm to carry out various design optimisation tasks. In principle, any optimization problems that can be solved by genetic algorithms and particle swarm optimisation can also be solved by the Firefly Algorithm. For simplicity to demonstrate its effectiveness in realworld optimisation, we use the FA to find the optimal solution of the standard but quite difficult pressure design optimisation problem.

Pressure vessels are literally everywhere such as champagne bottles and gas tanks. For a given volume and working pressure, the basic aim of designing a cylindrical vessel is to minimize the total cost. Typically, the design variables are the thickness $d_{1}$ of the head, the thickness $d_{2}$ of the body, the inner radius $r$, and the length $L$ of the cylindrical section (Coello 2000, Cagnina et al 2008). This is a well-known test problem for optimization and it can be written as

$$
\begin{aligned}
\operatorname{minimize} & f(\mathbf{x})=0.6224 d_{1} r L+1.7781 d_{2} r^{2} \\
+ & +3.1661 d_{1}^{2} L+19.84 d_{1}^{2} r
\end{aligned}
$$

subject to the following constraints

$$
\begin{aligned}
& g_{1}(\mathbf{x})=-d_{1}+0.0193 r \leq 0 \\
& g_{2}(\mathbf{x})=-d_{2}+0.00954 r \leq 0 \\
& g_{3}(\mathbf{x})=-\pi r^{2} L-\frac{4 \pi}{3} r^{3}+1296000 \leq 0 \\
& g_{4}(\mathbf{x})=L-240 \leq 0
\end{aligned}
$$

The simple bounds are

$$
0.0625 \leq d_{1}, d_{2} \leq 99 \times 0.0625
$$

and

$$
10.0 \leq r, \quad L \leq 200.0 .
$$

Recently, Cagnina et al (2008) used an efficient particle swarm optimiser to solve this problem and they found the best solution

$$
f_{*} \approx 6059.714,
$$

at

$$
\mathbf{x}_{*} \approx(0.8125,0.4375,42.0984,176.6366) .
$$

This means the lowest price is about $\$ 6059.71$.

Using the Firefly Algorithm, we have found an even better solution with 40 fireflies after 20 iterations, and we have obtained

$$
\mathbf{x}_{*} \approx(0.7782,0.3846,40.3196,200.0000)^{T},
$$

with

$$
f_{\min } \approx 5885.33
$$

which is significantly lower or cheaper than the solution $f_{*} \approx 6059.714$ obtained by Cagnina et al (2008).

This clearly shows how efficient and effective the Firefly Algorithm could be. Obviously, further applications are highly needed to see how it may behave for solving various tough engineering optimistion problems.

\section{Conclusions}

We have successfully used the Firefly Algorithm to carry out nonlinear design optimisation. We first validated the algorithms using some standard test functions. After designing some new test functions with singularity and stochastic components, we then used the FA to solve these unconstrained stochastic functions. We also applied it to find a better global solution to the pressure vessel design 
optimisation. The optimisation results imply that the Firefly Algorithm is potentially more powerful than other existing algorithms such as particle swarm optimisation.

The convergence analysis of metaheuristic algorithms still requires some theoretical framework. At the moment, it still lacks of a general framework for such analysis. Fortunately, various studies started to propose a feasible measure for comparing algorithm performance. For example, Shilane et al (2008) suggested a framework for evaluating statistical performance of evolutionary algorithms. Obviously, more comparison studies are highly needed so as to identify the strength and weakness of current metaheuristic algorithms. Ultimately, even better optimisation algorithms may emerge.

\section{References}

[1] Baeck, T., Fogel, D. B., Michalewicz, Z. (1997) Handbook of Evolutionary Computation, Taylor \& Francis.

[2] Cagnina, L. C., Esquivel, S. C., Coello, C. A. (2008) 'Solving engineering optimization problems with the simple constrained particle swarm optimizer', Informatica, 32, 319-326 (2008).

[3] Coello, C. A. (2000) 'Use of a self-adaptive penalty approach for engineering optimization problems', Computers in Industry, 41, 113-127.

[4] Deb, K. (1995) Optimisation for Engineering Design, Prentice-Hall, New Delhi.

[5] Dorigo, M. and Stützle, T. (2004) Ant Colony Optimization, MIT Press.

[6] Gazi, K., and Passino, K. M. (2004) 'Stability analysis of social foraging swarms', IEEE Trans. Sys. Man. Cyber. Part B - Cybernetics, 34, 539-557.

[7] Goldberg, D. E. (1989) Genetic Algorithms in Search, Optimisation and Machine Learning, Reading, Mass.: Addison Wesley.

[8] Holland, J. H. (1975) Adaptation in Natural and Artificial Systems, University of Michigan Press, Ann Arbor.

[9] Kennedy, J. and Eberhart, R. C. (1995) 'Particle swarm optimization', Proc. of IEEE International Conference on Neural Networks, Piscataway, NJ. pp. 1942-1948.

[10] Kennedy J., Eberhart R., Shi Y. (2001), Swarm intelligence, Academic Press.

[11] Lukasik, S. and Zak, S. (2009) 'Firefly algorithm for continuous constrained optimization tasks', ICCCI 2009, Lecture Notes in Artificial Intelligence (Eds. N. T. Ngugen, R. Kowalczyk, S. M. Chen), 5796, pp. 97-100.

[12] Shilane, D., Martikainen, J., Dudoit, S., Ovaska, S. J. (2008) 'A general framework for statistical performance comparison of evolutionary computation algorithms', Information Sciences: an Int. Journal, 178, 2870-2879.

[13] Yang, X. S. (2005) 'Biology-derived algorithms in engineering optimization', in Handbook of Bioinspired Algorithms and Applications (eds S. Olarius \& A. Y. Zomaya), Chapman \& Hall / CRC.

[14] Yang, X. S. (2008) Nature-Inspired Metaheuristic Algorithms, Luniver Press, UK.

[15] Yang, X. S. (2009) 'Firefly algorithms for multimodal optimization', in: Stochastic Algorithms: Foundations and Appplications (Eds O. Watanabe and T. Zeugmann), SAGA 2009, Lecture Notes in Computer Science, 5792, Springer-Verlag, Berlin, pp. 169-178. 